Stattdessen wurde die Innenpolitik in der zweiten Legislaturperiode vom wachsenden Reformdruck in der Arbeitsmarkt- und Sozialpolitik dominiert. Auf diesen Feldern waren überraschende Politikwechsel auszumachen, wie die Analysen von Josef Schmid (Arbeitsmarkt und Beschäftigung) und Manfred G. Schmidt (Sozialpolitik) vor Augen führen. Die Abkehr vom Prinzip der Statussicherung bewerten die Herausgeber als paradigmatischen Wandel. Den stärker marktliberal orientierten Kurs, der mithilfe „verkehrter Allianzen“ durchgesetzt worden sei, führen sie auf einen Strategiewechsel der Führungsspitze zurück. Als eine wesentliche Ursache für auftretende Implementationsprobleme kennzeichnen sie sachfremde Entscheidungen nach der Logik des Parteienwettbewerbs, wie etwa bei der Reform der Arbeitsagentur. Defizite treten auch in anderen untersuchten Politikfeldern zutage; allen voran bildete die Finanzpolitik und fehlgeschlagene Haushaltskonsolidierung (Uwe Wagschal) eine „Achillesferse“ der Regierungskoalition. Kritisch fällt die Bilanz auch in der von Interessenverbänden maßgeblich beeinflussten Gesundheitspolitik aus (Nils Bandelow und Anja Hartmann).

Ein höheres Maß an Kontinuität sehen Egle und Zohlnhöfer auf außenpolitischem Gebiet. Sie stützen sich dabei auf die Beiträge von Gunther Hellmann zur Außen- und Sicherheitspolitik und Tobias Ostheim zur Europapolitik. Dass sich die rot-grüne Koalition hier von Anfang an dramatischen Herausforderungen zu stellen hatte, zeigte bereits der KosovoEinsatz. Den Kulminationspunkt bildeten die Anschläge vom 11. September 2001, die zum Afghanistaneinsatz und aufgrund der Irakfrage schließlich zur Krise des transatlantischen Verhältnisses führten. Obwohl das Prinzip des Multilateralismus hierdurch gefährdet wurde, werten die Herausgeber das Nein zum Irakkrieg als Bestätigung rot-grüner (Friedens-)Politik. Die gleichzeitig „forcierte Renaissance nationaler Interessenpolitik“ (S. 516) erscheint hingegen als Handschrift des Kanzlers.

Eines vermag das Buch auch Skeptikern deutlich zu machen: Rot-grün hat Spuren hinterlassen, in materiellen Politikbereichen ebenso wie im deutschen Parteiensystem. Schließlich erkaufte die Bundesregierung ihre Reformerfolge zu einem hohen Preis, nämlich dem Mehrheitsverlust und dem Erstarken der Linkspartei.PDS. Dies wird den Parteienwettbewerb womöglich nachhaltiger verändern, als auf den ersten Blick ersichtlich ist. Aufgrund des Spektrums substanzieller Beiträge wie auch des reichen Datenmaterials ist der Band unverzichtbar für alle, die sich einen fundierten Überblick über Leistungen und Versagen der Regierungspolitik der letzten Jahre verschaffen wollen.

Manuela Glaab

\title{
Kooperation und Konsens bei der Normsetzung
}

Becker, Florian: Kooperative und konsensuale Strukturen in der Normsetzung, Mohr-Siebeck, Tübingen 2005, 828 Seiten, € 139,-.

Mit zunehmender Aufgabenbreite schwinde die Aufgabentiefe, mit wachsender Interventionsneigung sinke die Steuerungsfähigkeit des Staates. Sind solche Erkenntnisse mittlerweile fast schon von der opinio communis zum locus communis der Staatswissenschaften gekippt, 
so weist das Werk des Juristen Florian Becker einen Weg, auf dem sich Staatlichkeit weiterhin sogar neue Betätigungsfelder erschließen könnte, ohne dass sie an Tiefe, Genauigkeit und Wirksamkeit ihrer Steuerungsleistung verliert: Es ist der kooperative Staat, den als Ideal bereits seit den ausgehenden 1970er Jahren in Deutschland propagiert, der Autor zum Gegenstand seiner Studie macht. Er setze Normen kooperativ und konsensual zusammen mit den als vorrangigen Normadressaten betroffenen Partikularinteressen. Prototyp solcher Formen der Politikgestaltung ist der so genannte Atomkonsens, mit dem sich die vormalige von Sozialdemokraten und Grünen gebildete Bundesregierung bei der Novellierung des Atomgesetzes der Akzeptanz unmittelbar betroffener Interessen versicherte. Becker macht den Leser aber auch mit anderen Fällen kooperativer Normsetzung bekannt: Der Solidarbeitrag forschender Arzneimittelhersteller und das „Kraft-Wärme-Kopplungs-Gesetz“ bilden für ihn Beispiele, mit denen die Regierung von Gerhard Schröder einem neuen Regelungsmodus zum Durchbruch verholfen habe. Dieser ist Ausdruck eines Wandels staatlichen Handelns von hierarchischer Befehlsförmigkeit zu kooperativer Verhandlungsförmigkeit, der sich - von Sozialwissenschaftlern als Tatsache beobachtet, von Staatsrechtlern als Ideal postuliert grundsätzlich seit fast einer Generation in der westlichen Welt zu vollziehen beginnt.

Die entscheidende Frage an verhandlungsgestützte und konsensuale Normsetzungsabreden ist in einer parlamentarischen Demokratie selbstverständlich darin zu suchen, inwieweit eine Regierung verbindliche Aussagen über Gesetzgebung treffen oder gar das Parlament binden kann: Sie kann dies, wie Becker zeigt, nicht, was die politische Natur solcher Vereinbarungen offenbart. Könne sich die Bundesregierung rechtlich nur zum Gebrauch ihres Initiativrechts verpflichten, so sei indes das politische Dual von Opposition und Mehrheit und nicht mehr dasjenige von Legislative und Exekutive entscheidend. Freilich ist dafür bezeichnend, was Becker daran veranschaulicht, dass eine außerparlamentarische Vereinbarung wie der „Atomkonsens“ im Anhang detaillierte Regelungen hinsichtlich der weiteren Restlaufzeiten der deutschen Kernkraftwerke enthält. Bisweilen werden Übereinkommen sogar als Vergleiche vom Bundesverfassungsgericht vermittelt. Diese Entwicklung veranschaulicht Becker am Falle der geplanten Einführung des Unterrichtsfaches Leben, Ethik und Religionskunde in Brandenburg. Gegen inhaltliche Zusagen der Landesregierung hätten Beschwerdeführer ihre Einsprüche beim Bundesverfassungsgericht zurückgezogen: Leider geht aus Beckers Sachverhaltsschilderung nicht klar hervor, wer die „Beschwerdeführer, die ihren Antrag nicht zurückgenommen hatten“, sind (S. 189): Tatsächlich handelt es sich nicht nur um Beschwerdeführer, die in den vom Gericht moderierten Pakt eingewilligt beziehungsweise zumindest ihre Verfassungsbeschwerden in mündlicher Verhandlung zurückgezogen hatten, sondern auch um solche, die ihre Beschwerde eindeutig aufrechterhalten hatten. Der „Angriffsgegenstand“ sei „entfallen“, so lautete das Urteil des Ersten Senats ${ }^{1}$. Indem er dies verschweigt, wird vom Verfasser jedoch ein entscheidendes Problem in diesem Zusammenhang nicht klar herausgearbeitet, nämlich dasjenige dissentierender Partikularinteressen: Der Staat kann eben nicht ohne weiteres durch derartiges Vorgehen Steuerungstiefe bei fortgesetzter Steuerungsbreite zurückgewinnen, was Becker aber als realistisches Ziel ausgibt. Vielmehr droht er, Partikularinteressen, die sich wiederum innerhalb betroffener Partikularinteressen bilden, mit seiner ganzen arbiträren Macht zu sanktionieren und sich somit weiter partikularisieren zu lassen. 
Das opus magnum des mittlerweile im schottischen Aberdeen lehrenden Verfassungsjuristen beleuchtet mit einem methodisch breit ausgebildeten Instrumentarium letztlich die einschlägigen Steuerungsdilemmata sich immer weiter funktional differenzierender Gesellschaften, aber eine wirkliche Lösung chronischer Staatsüberforderung kann auch er nicht bieten. Am ehesten sei noch das deutsche Staatskirchenrecht als ein archetypisches Referenzgebiet ausgewiesen. Aber gerade der Charakter von Konkordaten als völkerrechtlichen Verträgen, die mit dem Vatikan geschlossen werden, unterscheidet sich kategorial von Abreden, die mit Privaten oder auch mit Selbstverwaltungsorganen betroffener Partikularinteressen hinsichtlich künftiger Gesetzgebung getroffen werden.

Politisches Recht beschreibt ein äußerst fluides Medium: Nur wenige Monate, nachdem das Buch von Becker erschienen war, sollte sich bekanntlich die Frage stellen, was aus dieser im Einklang mit Betroffenen vertraglich vereinbarten Politik unter der neuen Regierung werden sollte. Nicht nur die Große Koalition, sondern auch die Vertreter der Partikularinteressen müssen sich angesichts der Langfristigkeit der getroffenen Regelungen entscheiden, welches weitere Vorgehen ihnen mehr nützt. Somit wird vor allem das Problem der Kernenergie bislang weitgehend dilatorisch behandelt. Es könnte sich als weitsichtiger erweisen, die Vereinbarungen der Vorgängerregierung unangetastet zu lassen, als auf eine Revision des Konsenses zu pochen. Was das Rechtliche anbetrifft, so votiert Becker ohnehin für eine Selbstbindung der Politik, die sich im Zweifel selbst auf das Parlament erstrecken soll. Die Kernenergie ist hierbei nur Menetekel zukünftiger Probleme von Technik und Forschung, die immer stärker Eigendynamik und Eigengesetzlichkeit in ihrer zeitlichen Planung folgen und sich nicht in das Prokrustesbett von Legislaturperioden pressen lassen. Droht technisch-wissenschaftlicher Fortschritt, mit demokratischer Staatsverfassung gar unverträglich zu werden?

Ob der kooperative Staat als verheißungsvolle via regia zu einer Renaissance staatlicher Steuerung führen oder als neuer Nachtwächterstaat einen trüben Holzweg in eine entstaatlichte Postmoderne darstellen wird, bleibt indes das Geheimnis der Zukunft.

Daniel Hildebrand

\section{Das neue deutsche Regierungssystem}

Sturm, Roland und Heinrich Pehle: Das neue deutsche Regierungssystem. Die Europäisierung von Institutionen, Entscheidungsprozessen und Politikfeldern in der Bundesrepublik Deutschland, 2., aktualisierte und erweiterte Auflage, VS Verlag für Sozialwissenschaften, Wiesbaden 2005, 373 Seiten, $€ 19,90$.

Es gehört heute zum Bestand gesicherter politikwissenschaftlicher Erkenntnis, dass die Europäische Union einen immer stärkeren Einfluss auf nationalstaatliche Politikprozesse ausübt. Durch eine stetig wachsende Zahl europäischer Richtlinien und Verordnungen, die in nationales Recht überführt werden müssen, hat die EU eine erhebliche agenda settingMacht bekommen. Daher ist der Begriff der Europäisierung zu einem in der Politikwissenschaft gängigen, allerdings auch immer unbestimmteren Konzept geworden, das die fort- 